\title{
LC/MS and LC/MS/MS Determination of Protein Tryptic Digests
}

\author{
Eric C. Huang and Jack D. Henion \\ Drug Testing and Toxicology, Cornell University, Ithaca, New York, USA
}

\begin{abstract}
Tryptic digests were analyzed by means of online microbore liquid chromatography combined with mass spectrometry (LC/MS) for some common proteins. Following conventional enzymatic digestion with trypsin, the freeze-dried residues were dissolved in highperformance liquid chromatography (HPLC) eluent and subjected to gradient reversed-phase microbore HPLC separation with mass spectrometric detection. The latter was done in the full-scan single or tandem (MS/MS) mass spectrometry mode. The formation of gas-phase ions from dissolved analytes was accomplished at atmospheric pressure by pneumatically assisted electrospray (ion spray) ionization. This produced field-assisted ion evaporation of dissolved ions, which could then be mass-analyzed for molecular mass or structure. In the full-scan LC/MS mode, the masses for the peptide fragments in the tryptic digests can be determined as either their singly or multiply charged ions. When the molecular weights of the peptides lie outside the mass range of the mass spectrometer, the multiply charged feature of these experimental conditions still provides reliable molecular weight determinations. In addition, collision-activated dissociation (CAD) on selected peptide precursor ions provides online LC/MS/MS sequence information for the tryptic fragments. Results are shown for the tryptic digests of horse heart cytochrome $c$, bovine $\beta$-lactoglobulin A, and bovine $\beta$-lactoglobulin B. (J Am Soc Mass Spectrom 1990, 1, 158-165)
\end{abstract}

$\mathrm{T}$ The combination of high-performance liquid chromatography (HPLC) and mass spectrometry (LC/MS) remains a desirable goal. Although significant advances have occurred over the last fifteen years $[1,2]$, the use of routine online LC/MS capability is common in only a few laboratories worldwide. Most of the successful problem-solving applications, however, focus on so-called small, moderately polar molecules whose molecular weights are typically less than 500. Neither the thermospray $[3,4]$ nor particle beam [5] LC/MS interfaces have shown much promise for high-sensitivity determinations of the more challenging biomedical molecules such as peptides and proteins. Fast atom bombardment (FAB) in the continuous flow FAB $[6,7]$ mode has recently shown considerable improvement, but the chromatographic flow rates must be maintained below approximately $5 \mu \mathrm{L} / \mathrm{min}$ by using either open tubular [8] or packed capillary columns [9] unless a postcolumn flow split is employed. Although preliminary flow FAB microLC/MS results look encouraging, the use of routine reversed-phase separations of real samples has not yet appeared.

Currently there is considerable interest in the mass spectrometric characterization of biomolecules. Analytical biochemistry has moved slowly from the use of slab electrophoretic techniques for separations and

Address reprint requests to Jack D. Henion, Drug Testing and Toxicology, Cornell University, 925 Warren Drive, Ithaca, NY 14850. ultracentrifugation for molecular mass determinations. Analytical biotechnology, too, has brought about a need for improved analytical techniques for the characterization of primary through quaternary structures of proteins [10].

High-performance liquid chromatography has enjoyed considerable acceptance in the area of biomolecule sample separations [11]. Researchers and commercial suppliers of modern bioactive compounds rely heavily on this technique for both analytical and preparative separations. Conventional UV and fluorescence detectors, however, have limitations. They lack specificity and sensitivity, so structural characterization of mixture components must be gained by alternative techniques. In effect, HPLC provides only separation of mixture components and an indication that something elutes at a particular retention time. It is clearly desirable to have separation with online specific and sensitive detection [12].

A combination of HPLC and mass spectrometry (LC/MS) provides these attributes. Although the mass spectrometer imposes some limitations on HPLC eluent and modifiers (buffers), for the most part acceptable LC/MS analyses of biological mixtures can be accomplished. Gradient reversed-phase capability is typically required with careful selection of HPLC column packings, hardware, and eluent modifiers. As this paper will show, however, good analytical separations and sensitive detection can be achieved with mass spectrometric detection. 
A perceived limitation of mass spectrometry in the past has been its mass range. Most quadrupole mass spectrometers have mass ranges from 1000 to $4000 \mathrm{u}$, whereas some magnetic and Fourier transform (FT) mass spectrometers provide greater capability approaching 20,000 u. Only time-of-flight (TOF) mass spectrometers have reported capability approaching $100,000 \mathrm{u}$ [13], but this is accomplished with peak widths at half-height of several thousand mass units. Of course, none of these technologies impresses the protein chemist interested in biomolecules with molecular weights as high as several million units.

An exciting breakthrough in accessing the higher molecular weight ranges with conventional mass spectrometers occurred with the work of Fenn and coworkers $[14,15]$. Their report of multiple charging for high molecular weight compounds from electrospray ionization appears to have expanded our view of mass spectrometry. Other reports on the continuous slow infusion of peptide and protein solutions by ourselves $[16,17]$ and Smith and co-workers [18] corroborated the exciting potential this new capability has for biochemistry and biotechnology. In our work the 16,950-u molecular mass of bovine $\beta$-lactoglobulin A was determined to within less than $1 \mathrm{u}$ on a quadrupole mass spectrometer with a mass range of only $1400 \mathrm{u}$ [16], whereas Smith et al. [19] reported the molecular mass of $1.33 \times 10^{5} \mathrm{u}$ for the bovine serum albumin dimer.

All the multiple charging of high-mass biomolecules reported to date has involved the infusion of singlecomponent solutions. Obviously the mass spectrometric analysis of a complex mixture such as an enzymatic digest or a fermentation broth would benefit from some prior chromatographic separation. It is well known that HPLC is currently the most common technique for accomplishing this task, and the generation of tryptic maps [20], for example, is considered a very useful bioanalytical tool.

We propose that the use of online mass spectrometric detection with HPLC is a convenient and preferred approach to conventional HPLC tryptic mapping. The pneumatically assisted electrospray (ion spray) LC/MS interface [21] implemented with an atmospheric pressure ionization (API) ion source provides continuous, stable, and sensitive detection of peptides and proteins separated online by microbore HPLC. In one 30-min gradient HPLC analysis, the molecular mass for each tryptic fragment in the mixture can be determined to within $1 \mathrm{u}$. Once the identity of the molecular ion for each peptide is known, a subsequent HPLC analytical determination in combination with tandem mass spectrometry (LC/MS/MS) can provide sequence information for each tryptic fragment in the HPLC total ion current (TIC) chromatogram. This analytical capability should offer considerable help for the solution of important problems in biochemistry and biotechnology. We present results that demonstrate this capability using tryptic digests of horse heart cytochrome $c$, bovine $\beta$-lactoglobulin A, and bovine $\beta$-lactoglobulin B.

\section{Experimental}

\section{Materials}

The proteins used in this study were bovine $\beta$-lactoglobulin A, bovine $\beta$-lactoglobulin $\mathrm{B}$, and horse heart cytochrome $c$. The enzyme that was used to digest the protein was trypsin treated with L-1-tosylamide-2-phenylethyl chloromethyl ketone (TPCK). These materials were purchased from Sigma Chemical Co. (St. Louis, MO) and were used as received without any treatment prior to the digestion procedure. Sequencing-grade trifluoroacetic acid (TFA) was obtained from Aldrich Chemical Co. (Milwaukee, WI), HPLC-grade acetonitrile was obtained from J.T. Baker Chemical Co. (Phillipsburg, NJ), and HPLCgrade water was from Fisher Scientific (Rochester, NY).

\section{Enzymatic Digestion}

Bovine $\beta$-lactoglobulin A, bovine $\beta$-lactoglobulin $\mathrm{B}$, and horse heart cytochrome $c$ were digested with TPCK-treated trypsin for $16-20 \mathrm{~h}$ at $37^{\circ} \mathrm{C}$ with a substrate-to-enzyme ratio of $50: 1(\mathrm{w} / \mathrm{w})$ in $50 \mathrm{mM}$ ammonium bicarbonate buffer solution at $\mathrm{pH} 8.5$ (the $\mathrm{pH}$ of the buffer solution was adjusted with $1 \mathrm{M}$ ammonium hydroxide). The digestion solution was then lyophilized and redissolved in water containing $3 \mathrm{mM}$ TFA with an approximate sample concentration of 0.5 , or $1 \mathrm{mg}$ protein per milliliter solvent. A sample size of $5 \mu \mathrm{L}$ was typically injected on-column unless otherwise stated.

\section{Microbore Liquid Chromatography}

The preferred microbore LC column used for all protein tryptic digest separations was $1 \mathrm{~mm}$ in internal diameter, $10 \mathrm{~cm}$ long, and packed with $5-\mu \mathrm{m} \mathrm{LC}-308$ packing material (bonded with C-8 stationary phase, $300 \AA$ pore size) provided by Supelco (Supelco, Inc., Bellefonte, PA). The micro LC system consisted of a Brownlee Labs Micropump (Santa Clara, CA) and was used without any modification. The injection of sample was accomplished with a Model 9125 biocompatible syringe-loading sample injector with a $5-\mu \mathrm{L}$ external sample loop (Rheodyne, Cotati, CA). The mobile phase was composed of $3 \mathrm{mM}$ TFA/ $\mathrm{H}_{2} \mathrm{O}$ as solvent $\mathrm{A}$ and $3 \mathrm{mM}$ TFA $/ \mathrm{CH}_{3} \mathrm{CN}$ as solvent $\mathrm{B}$. All separations were accomplished by applying a linear solvent gradient from $5 \%$ solvent $B$ to $70 \%$ solvent $B$ in a time period of $60 \mathrm{~min}$. With this gradient program, the LC run times were typically within $40 \mathrm{~min}$. The column flow was maintained at $40 \mu \mathrm{L} / \mathrm{min}$ throughout these experiments. The exit of the analytical column was connected directly to the pneumatically assisted electrospray interface. There was no presplit (before the column) or postsplit (after the column, before entering the ion spray interface) of the mobile phase. 


\section{Mass Spectrometry}

A Sciex (Thornhill, Ontario, Canada) TAGA 6000E triple quadrupole mass spectrometer equipped with an API source was used. The interface for coupling the microbore LC system with API/MS was a pneumatically assisted electrospray (ion spray) interface that was constructed in-house. Details of the interface construction were described previously [21]. The LC/MS interface sprayer can be floated at $\pm 3 \mathrm{kV}$ potential. The polarity of this potential is dependent upon the operational mode (i.e., positive ion or negative ion detection) of the mass spectrometer. In the present study the mass spectrometer was operated under positive ion mode of detection for protonated peptides. Ions generated from the ion spray interface via ion evaporation mechanisms were sampled into the mass spectrometer by a potential difference (typically 2.5 $\mathrm{kV}$ ) set between the sprayer and the sampling orifice. The sampling orifice was a $100-\mu \mathrm{m}$-diameter hole at the end of the conical nozzle. Ultrapure nitrogen (Linde Specialty Gases, Danbury, CT) was applied at the atmospheric side of the skimmer to minimize solvent clusters and particulate matter entering the mass spectrometer. For online LC/MS experiments, the first quadrupole (Q-1) was scanned from $\mathrm{m} / \mathrm{z} 350$ to $\mathrm{m} / \mathrm{z} 1400$ in $10 \mathrm{~s}$ with a scan step of $1 \mathrm{u}$. For MS/MS, ultrapure argon (AIRCO, The BOC Group, Inc., Murray Hill, $\mathrm{NJ}$ ) was used as the collision gas and was introduced into the collision cell (Q-2) at a target gas thickness of $200 \times 10^{12}$ atoms $/ \mathrm{cm}^{2}$. The collision energy was controlled by the Q-2 offset voltage. A typical Q-2 offset voltage was $-20 \mathrm{~V}$ in this work unless otherwise stated. The product ion mass spectra were acquired by scanning the third quadrupole $(\mathrm{Q}-3)$ while $\mathrm{Q}-1$ was set for transmitting the precursor ion. The Q-3 offset was programmed to provide optimum ion transmission efficiency.

\section{Results and Discussion}

One of the most important matters to address in this work is the proper choice of HPLC hardware and column packing. Our early attempts were frustrated by unsatisfactory chromatograms with both UV and mass spectrometric detection. An example is shown in Figure 1, where the same tryptic digest of bovine $\beta$-lactoglobulin A was analyzed by LC/MS using two different $C-8,1 \mathrm{~mm} \times 100 \mathrm{~mm}$, packedmicrobore columns. The eluent flow was maintained at $40 \mu \mathrm{L} / \mathrm{min}$ with a gradient from $5 \%$ B to $70 \%$ B in $60 \mathrm{~min}$. Five microliters of the tryptic digest sample whose concentration was $1 \mathrm{mg} / \mathrm{mL}$ in eluent was injected, while the total microbore HPLC effluent was directed to the LC/MS interface. The only differences between column A and column B in Figure 1 were the vendor and presumably the treatment of the C-8 bonded silica. As can be seen from this figure, column $B$ provided much greater selectivity for the tryptic peptides and a better chromatographic peak shape than column A. Therefore, all the remaining data described in this work were obtained with column B.

Because tryptic mapping by HPLC with UV or fluorescence [20] detection has become quite popular, we investigated the utility of mass spectrometry for this purpose. Figure $1 b$ and $c$ show the microbore LC/MS TIC chromatogram obtained from the analysis of the tryptic digests of bovine $\beta$-lactoglobulin $\mathrm{A}$ and bovine $\beta$-lactoglobulin B with the use of column B and a reversed-phase gradient. Inspection of these two chromatograms reveals many similarities yet some distinct differences. For example, the chromatographic peak at about $8 \mathrm{~min}$ in Figure $1 \mathrm{~b}$ is absent in Figure 1c. In contrast, the chromatographic peak centered at about 14 $\min$ in Figure $1 \mathrm{c}$ is absent in Figure $1 \mathrm{~b}$. The differences and similarities typically noted in tryptic mapping using HPLC with conventional detectors are considered
Figure 1. Full-scan LC/MS TIC profile from the analysis of a protein tryptic digest using two related but different HPLC column packings. Both columns had dimensions of $1 \mathrm{~mm} \times 100 \mathrm{~mm}$ and were packed with $5-\mu \mathrm{m}$ particles of $\mathrm{C}-8$ bonded silica. (a) Bovine $\beta$-lactoglobulin A tryptic digest using column $A$, which was packed with $C-8$; (b) same as in (a) except using column $B$ packed with LC-308 and obtained from Supelco Inc.; (c) $\beta$ lactoglobulin B tryptic digest using column B. HPLC conditions: linear gradient from $5 \%$ B to $60 \% \mathrm{~B}$ in $60 \mathrm{~min}$ (actual chromatographic run time was $40 \mathrm{~min}$ ). The mass scan was from $\mathrm{m} / \mathrm{z} 300$ to $m / z 1400$ in $10 \mathrm{~s}$.

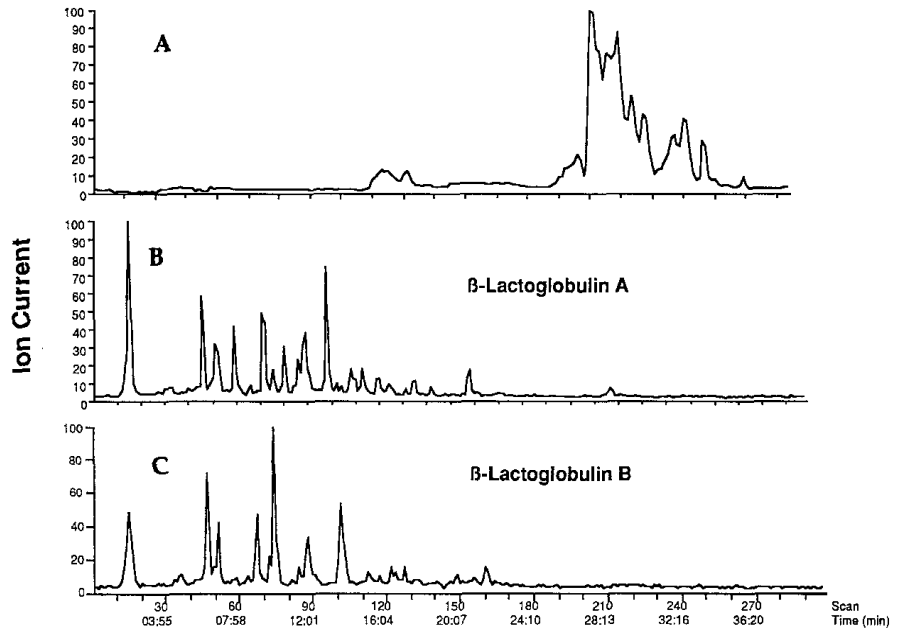


analytically useful. With LC/MS, however, the mass spectra and molecular weights for each component can be obtained from the same analytical determination. In this approach, the chromatographic peaks can be distinguished by their mass spectra rather than just by their retention time.

There were other important details to address to ensure satisfactory results from the LC/MS analysis of these biological mixtures. Although the so-called biocompatible injector was used in this work, we have no direct evidence that it provided superior performance. A recent report suggests there may be specific benefits when certain compounds are being handled [22], but such claims are often difficult to document. We chose to use this injector to minimize potential adsorptive and degradative losses but cannot attest to any particular advantages.

Perhaps the most important experimental details to address are precolumn and postcolumn plumbing connections. The minimization of extracolumn band broadening is particularly important in microbore HPLC. It is also important to make high-quality connections at all junctions and to minimize these junctions and the lengths of connecting tubing. All tubing had a nominal inside diameter of $100 \mu \mathrm{m}$, and an untreated fused-silica transfer line $(100 \mu \mathrm{m}$ i.d. $\times 20 \mathrm{~cm})$ was used to connect the column to the LCIMS interface.

The microbore LC/MS ion current profiles in Figure $2 \mathrm{a}$-e give some indication of the chromatographic integrity available from the system when all the important experimental details have been addressed. The microbore LC/MS TIC chromatogram obtained from the analysis of a tryptic digest of horse heart cytochrome $c$ by using column $B$ are shown in Figure 2e. Five microliters of the tryptic digest sample $(0.5 \mathrm{mg} / \mathrm{mL})$ dissolved in the mobile phase, which amounts to approximately 216 pmol of each peptide, was injected. Routine determinations of 100-150 pmol have been achieved [17], and it has been shown that low picomole detection should be possible [23].

Although all components are not completely resolved under these conditions, at least 20 compounds were detected. The extracted ion current profiles for components giving ions at $m / z 729,361,390$, and 1006 are shown in Figure 2a-d, respectively. These data readily indicate which chromatographic peaks in the TIC chromatogram (Figure 2e) correspond to these $\mathrm{m} / \mathrm{z}$ values, and their symmetry and relatively narrow widths suggest the quality of the HPLC separation. For example, the ion current profiles in Figure $2 b$ and $c$ for ions of $\mathrm{m} / \mathrm{z} 361$ and 390 are easily distinguished as being due to separate components compared to their unresolved presence in the TIC chromatogram. The components elute at about $13 \mathrm{~min}$ retention time. Although the TIC chromatogram shows these two components unresolved, their differing retention times and compositions are corroborated in Figure $2 b$ and $c$. Their corresponding mass spectra can be obtained and their
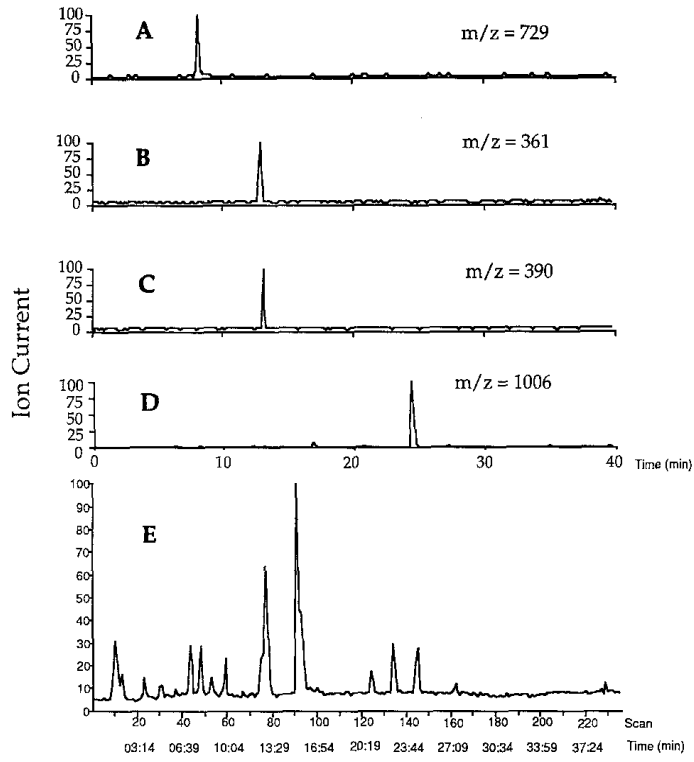

Figure 2. Full-scan LC/MS TIC profile (e) and extracted ion current profiles (a-d) obtained from the analysis of a tryptic digest of horse heart cytochrome $c$. Conditions same as for Figure 1.

molecular weights determined by using conventional background subtraction routines.

Not all of the 21 theoretical tryptic fragments expected from the tryptic digest of horse heart cytochrome $c$ are observed in Figure 2e. Because there is chemical noise resulting from solvent ion clusters below $m / z 350$, the lower limit of our scan range was limited to acquisition of ion current from $\mathrm{m} / \mathrm{z} 350$ to 1400 . Because there are ten predicted tryptic fragments from horse heart cytochrome $c$ whose molecular masses are lower than $350 \mathrm{u}$, we do not detect the smaller peptides under these scan conditions. All of the remaining eleven tryptic fragments are, however, detected, because their molecular masses are within the scanned mass range. Table 1 summarizes the contents of the tryptic digest of horse heart cytochrome $c$ and lists the eleven observed peptides along with their corresponding singly or doubly charged molecular ions.

Closer inspection of the microbore LC/MS TIC chromatogram shown in Figure 2e reveals the detection of over twenty chromatographic components that eluted within $40 \mathrm{~min}$. The mass spectrum for each of these components is available for inspection, and usually the molecular mass of each component can be easily determined because of the extremely mild ionization conditions provided by this mass spectrometric system. For example, Figure $3 a-d$ show the mass spectra for the chromatographic peaks eluting at 10, 13, 13.2, and 25 min in Figure 2a-d.

The molecular masses for these components can be readily determined from the corresponding abundant ions observed at $m / z$ 729, 361, 779, and 1006 in Figure 3a-d [17]. Because this LC/MS interface produces 
Table 1. Theoretical and observed mass of tryptic peptides of horse heart cytochrome $c$ from ion spray LC/MS

\begin{tabular}{|c|c|c|c|c|}
\hline Theoretical ${ }^{\mathrm{a}}$ & MW & Observed & $(M+H)^{+}$ & $(\mathrm{M}+2 \mathrm{H})^{2+}$ \\
\hline $\operatorname{Tp} 1$ & 546 & Tp 1 & 547 & $-b$ \\
\hline $\operatorname{Tp} 4$ & 634 & $\operatorname{Tp} 4$ & 635 & $-{ }^{b}$ \\
\hline Tp 5 & 1018 & Tp 5 & -0 & 570 \\
\hline Tp 8 & 1168 & Tp 8 & $-c$ & 585 \\
\hline Tp 10 & 1456 & Tp 10 & $-{ }^{\mathrm{b}}$ & 729 \\
\hline Tp 12 & 2010 & Tp 12 & $-{ }^{b}$ & 1006 \\
\hline Tp 14 & 678 & Tp 14 & 679 & $-\mathrm{b}$ \\
\hline Tp 15 & 779 & Tp 15 & $-c$ & 390 \\
\hline Tp 18 & 360 & Tp 18 & 361 & $-{ }^{b}$ \\
\hline Tp 19 & 964 & Tp 19 & $-c$ & 483 \\
\hline Tp 21 & 433 & Tp 21 & 434 & $-\mathrm{b}$ \\
\hline
\end{tabular}

a Horse heart cytochrome $c$ tryptic peptide fragments whose singly or doubly charged molecular ions lie within the $m / z$ 350-1400 range scanned during the course of these LC/MS experiments.

b Out of the mass spectrometry scan range (350-1400 u).

- Low ion abundance.

Note: All the expected tryptic peptides [11] are observed.

no fragment ions from these peptides, their abundant precursor ions are readily discernible. For the smaller peptides or for those that have only one basic amino acid residue, singly protonated, singly charged precursor ions usually predominate; this is observed, for example, for the T-18 fragment shown in Figure 3b. When these peptides have multiple basic sites (amino acids) or basic sites plus a terminal amino moiety, however, they often produce multiply protonated, multiply charged precursor ions [15-18].
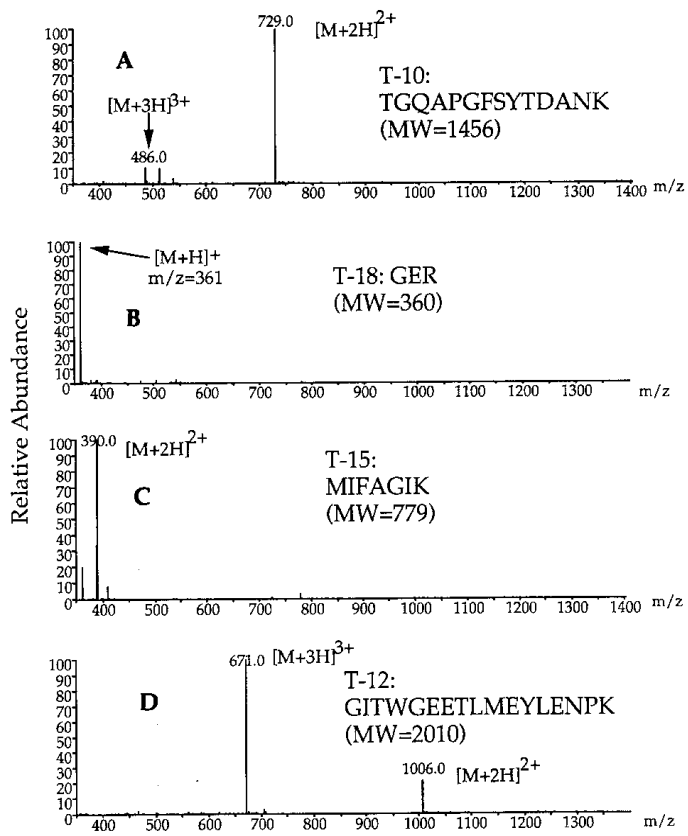

Figure 3. Full-scan ion evaporation mass spectra of (a) T-10, (b) $\mathrm{T}-18$, (c) T-15, and (d) T-12 peptides obtained from the tryptic digest of horse heart cytochrome $c$ (Figure 2).
The degree of multiple charging can be readily calculated [16], and hence one can determine the molecular masses of peptides that may lie outside the mass range of the mass spectrometer. An example of this is seen in Figure $3 \mathrm{~d}$, where the molecular mass of the T12 fragment is $2010 u$, or $610 u$ above the mass range of our quadrupole mass spectrometer. However, its doubly protonated, doubly charged ion at $m / z 1006$ and its triply protonated, triply charged ion at $m / z 671$ are readily detected. Molecular masses above the mass range of the mass spectrometer, therefore, can be readily determined for the components detected in a gradient microbore LC/MS analytical determination of such a tryptic digest.

When one has the molecular masses for components of interest in the LC/MS TIC chromatogram from a tryptic digest, the next logical step is to combine HPLC with MS/MS in an effort to gather sequence information from collision-activated dissociation (CAD). Online LC/MS/MS is, in fact, a natural combination with this LC/MS interface. The very mild ionization conditions produce mass spectra with most of the ion current in one or two ions. These may be selectively focused into the collision cell for CAD to gather sequence or structural information based on the formation of product ions.

The product ion full-scan TIC chromatograms shown in Figure 4 were obtained from the online microbore LC/MS/MS analysis of the tryptic digest of bovine $\beta$-lactoglobulin $\mathrm{A}$. The gradient microbore HPLC experimental conditions used were the same as described for Figure 1 . The T-8 singly protonated, singly charged precursor ion at $\mathrm{m} / \mathrm{z} 573$ was focused with Q-1 into the collision cell Q-2, and argon was used with a collision energy of $50 \mathrm{eV}$ (laboratory frame) and a collision gas thickness of $200 \times 10^{12}$ atoms $/ \mathrm{cm}^{2}$ for the CAD experiment shown in Figure 4a. Q-3 was scanned repetitively from $\mathrm{m} / \mathrm{z} 50$ to $\mathrm{m} / \mathrm{z} 1300$. The full-scan CAD 

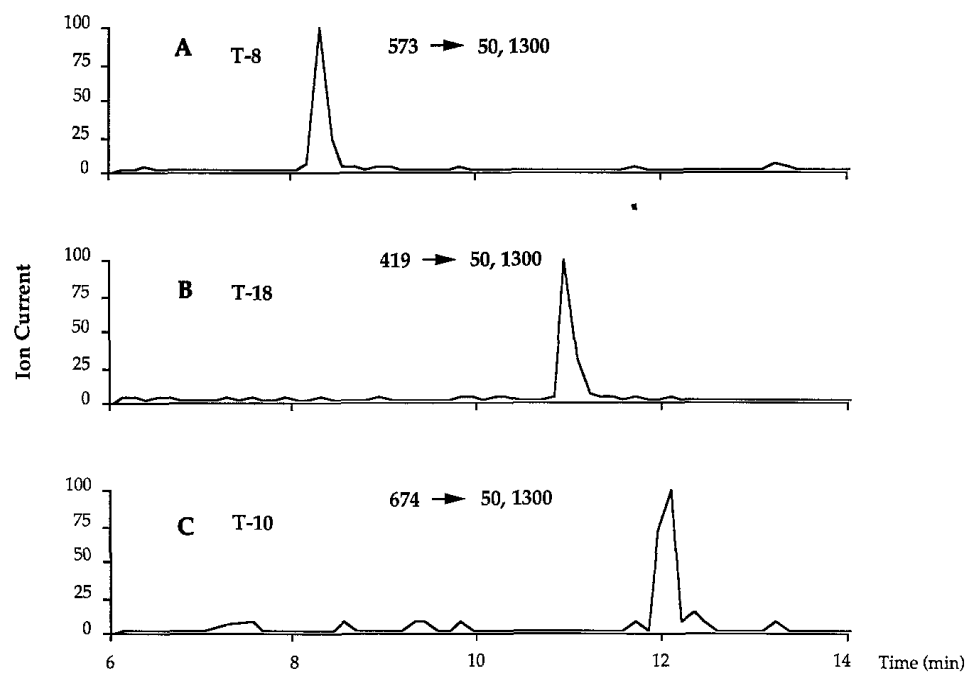

Figure 4. Full-scan LC/MS/MS (m/z 501300) product ion TIC chromatograms for the analysis of a tryptic digest of bovine $\beta$ lactoglobulin A. (a) CAD on $\mathrm{m} / \mathrm{z} 573$, T-8; (b) CAD on $\mathrm{m} / \mathrm{z} 419$, T-18; (c) CAD on $\mathrm{m} / \mathrm{z}$ 674, T-10. See text for LC/MS/MS experimental details.

product ion mass spectrum resulting from this experiment is shown in Figure 5. This mass spectrum was taken from the apex of the chromatographic peak centered at $8.3 \mathrm{~min}$ retention time in Figure $4 \mathrm{a}$. Although the singly protonated, singly charged precursor ion at $m / z 573$ remains as the most abundant in this 50$\mathrm{eV}$ spectrum, an abundance of structurally significant product ions is observed. These include the 1-4 fragments from the $\mathrm{A}, \mathrm{B}$, and $\mathrm{Y}^{\prime \prime}$ series of sequence ions [24]. The presence of each ion series in this CAD spectrum provides evidence for the corresponding amino acids in the peptide sequence. Although additional information would be needed for characterizing isomeric residues, sequence information from these product ion spectra should be possible and rules leading to the general use of such spectra for these purposes could be developed. Considerable effort is still required, however, to test these generalizations, and this work is under way.
The CAD mass spectrum for the doubly protonated, doubly charged precursor ion-product ion relationship shown in Figure $4 \mathrm{~b}$ is shown in Figure 6. The precursor ion at $m / z 419$ was focused with Q-1 into the collision cell, Q-2, and argon was used with a collision energy of $50 \mathrm{eV}$ (laboratory frame) and a collision gas thickness of $200 \times 10^{12}$ atoms $/ \mathrm{cm}^{2}$. Q-3 was scanned repetitively from $m / z 50$ to $m / z 1300$ in $10 \mathrm{~s}$. The online LC/MS/MS total product ion current profile from $\mathrm{m} / \mathrm{z} 50$ to $\mathrm{m} / \mathrm{z}$ 1300 resulting from CAD of the $\mathrm{m} / \mathrm{z} 419$ precursor ion for the analysis of the tryptic digest from bovine $\beta$ lactoglobulin $A$ is shown in Figure $4 b$. The CAD mass spectrum shown in Figure 6 was obtained from the chromatographic peak centered at $11.1 \mathrm{~min}$ retention time shown in Figure $4 \mathrm{~b}$.

Inspection of the $m / z 419$ product ion spectrum in Figure 6 shows an abundance of fragment ions including some ( $m / z 556,636,653$, and 766) above the massto-charge ratio range of the precursor ion. The pres-

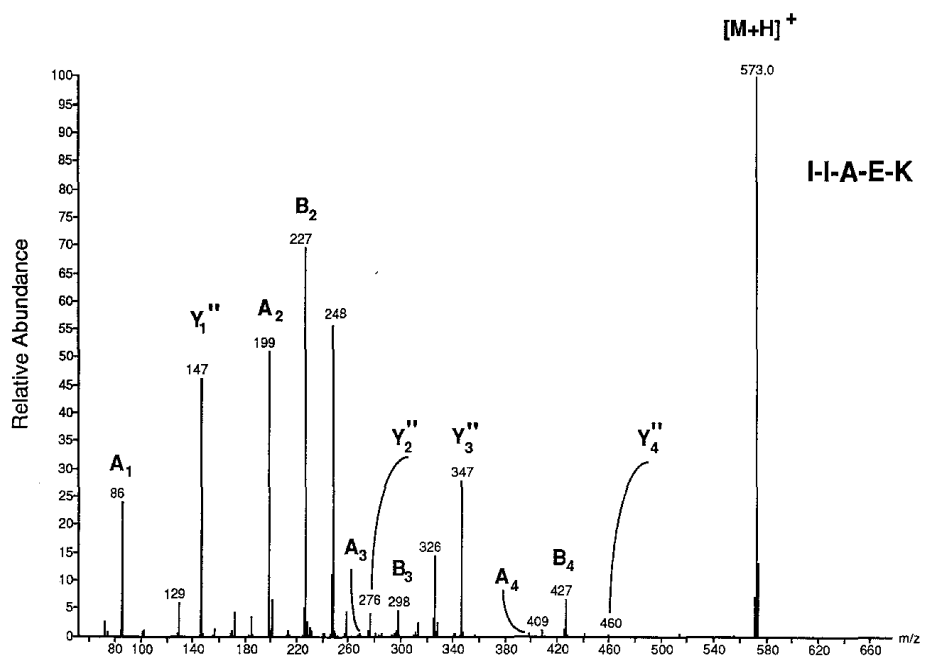

Figure 5. Full-scan CAD mass spectrum of a singly charged tryptic peptide (T-8) derived from $\beta$-lactoglobulin A (Figure 4a). For conditions, see Figure 4. 
Figure 6. Full-scan CAD mass spectrum of a doubly charged tryptic peptide (T-18) derived from bovine $\beta$-lactoglobulin $A$ (Figure $4 \mathrm{~b}$ ). For conditions, see Figure 4 and text.

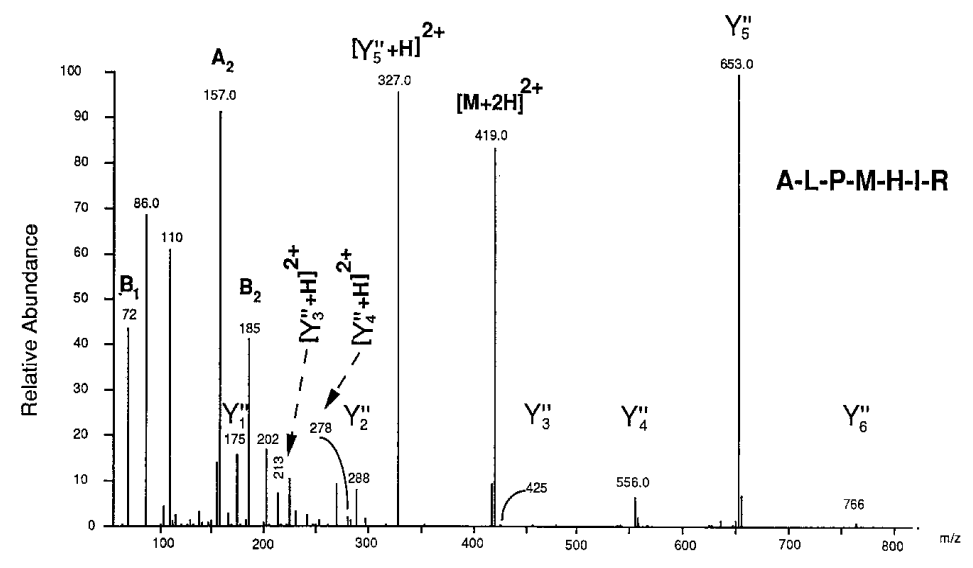

ence of product ions at a higher $m / z$ than the precursor ion is a unique feature for CAD of multiply charged ions. Although the interpretation of the CAD spectrum resulting from a doubly charged ion would be expected to be less straightforward than that described above, it is still possible to gather useful information from such data. Unlike the rather confusing information reported from CAD of precursor ions containing many charges [18], the product ion spectra resulting from doubly charged ions appear to be useful [25]. Inspection of Figure 6 reveals abundant singly charged ions at $m / z$ 653 and 157, for example, but also an abundant doubly charged fragment ion at $\mathrm{m} / \mathrm{z} 327$ with weaker doubly charged ions at $m / z 213$ and 278. These latter ions can complicate the interpretation of results, but knowledge of their likelihood can help clarify their origin.

The product ion mass spectra in Figures 5 and 6 reveal fragments corresponding to $\mathrm{A}, \mathrm{B}$, and $\mathrm{Y}^{\prime \prime}$ series ions [24]. On the basis of knowledge gained from FAB/MS/MS peptide sequencing from singly charged precursor ions, it has been suggested that the most commonly observed fragment series are $A, B, C^{\prime \prime}, Y^{\prime \prime}$, $Z$, and $Z$ " for positive ion spectra and $C$, " $X, Y,{ }^{\prime \prime} Y$, and $Z$ for negative ion spectra [26-29]. Our positive ion CAD spectra from both singly and doubly charged precursor ions indicate a complete fragment series of $A, B$, and $Y^{\prime \prime}$ ions. Further work is in progress to delineate the relevance of CAD product ion mass spectra to structural characterization of doubly charged ions such as those resulting from tryptic peptides.

Figure 6 also indicates an unusually abundant product ion at $m / z$ 327. Examination of possible CAD fragments from this heptapeptide derived from the bovine $\beta$-lactoglobulin A tryptic digest reveals no obvious association with the established amino acid sequence. Because the $m / z 419$ precursor ion was known to be doubly protonated and doubly charged, the $m / z 327$ fragment ion may be either singly or doubly charged. If the precursor ion were to be protonated at remote amino acid sites, the cleavage of an internal bond in the CAD process could yield two singly charged fragment ions. However, if one of these fragments con- tained a basic amino acid such as the histidine present in this T-18 fragment, then both the histidine and the amino terminus could be protonated, thus generating a doubly charged fragment ion. The additional proton in this doubly charged fragment ion would have to result from a rearrangement process to produce the observed $\left[\mathrm{Y}^{\prime \prime}+\mathrm{H}\right]^{2+}$ product ion. Thus we suggest that the abundant $m / z 327$ fragment ion results from the addition of a proton to the singly charged $Y_{5}^{\prime \prime}$ fragment ion. Further work on known peptides is needed to verify this speculation. Additional support for this observation would be provided by the presence of doubly charged fragments derived from $\mathrm{Y}_{3}^{\prime \prime}$ and $\mathrm{Y}_{4}^{\prime \prime}(\mathrm{m} / \mathrm{z}$ 425 and 556, respectively). This is corroborated by the presence of ions at $m / z 213$ and 278, respectively, in Figure 6.

FAB/MS/MS studies of peptides that contain a histidine residue show that $\mathrm{CAD}$ always gives an immonium ion at $m / z 110[30,31]$. We also see $m / z 110$ in Figure 6 . This is a convenient way to determine the presence of histidine in the peptide, although not its position in the peptide chain. The results described above for $\mathrm{CAD}$ of the doubly charged ion, however, also provide information on the position of the histidine via reading through the doubly charged $\mathrm{Y}^{\prime \prime}$ ions.

\section{Summary and Conclusions}

The use of LC/MS and LC/MS/MS for the characterization of protein tryptic digests can be a very powerful bioanalytical technique. A mass spectrometer equipped with a pneumatically assisted electrospray (ion spray) LC/MS interface with an API ion source can maintain good chromatographic integrity for reversedphase gradient HPLC separations of these digests at the low picomole level. In addition to the information afforded by conventional tryptic mapping using HPLC with UV or fluorescence detection, microbore LC/MS can assist in the identification of each peptide by providing its molecular mass. Peptides whose molecular masses lie beyond the mass range of the mass spec- 
trometer can be determined by calculating the number of charges exhibited by the precursor ions formed.

In addition to determining the molecular masses for the tryptic peptides, it is feasible to obtain amino acid sequence information by combining HPLC with online MS/MS. Once the precursor ions are known from the microbore LC/MS results, one can sequentially focus them into the collision cell in a subsequent online analysis to obtain the full-scan CAD mass spectra. Both singly and doubly charged precursor ions appear to provide meaningful structural information that can facilitate characterization of the peptide sequence. Although additional systematic work is necessary to develop this interpretation ability, it is anticipated that this approach to peptide and hence protein characterization will offer tremendous assistance to analytical biochemistry.

\section{Acknowledgments}

We thank Richard Ludwig of Supelco Inc. for providing the microbore C-8 column and Sciex for hardware and research support. We also thank Dr. T. Covey for assistance and helpful suggestions during the course of this work, and E.C.H. thanks the Eastman Kodak Company for financial support.

\section{References}

1. Arpino, P. J.; Guiochon, G. Anal. Chem. 1979, 51, 682A-701A.

2. Covey, T. R.; Lee, E. D.; Bruins, A. P.; Henion, J. D. Anal. Chem. 1986, 58, 1451A-1461A.

3. Garteiz, D. A.; Vestal, M. L. LC Mag. 1985, 3, 334-346.

4. Stachowiak, K.; Wilder, C.; Vestal, M. L.; Dyckes, D. F. J. Am. Chem. Soc. 1988, 110, 1758-1765.

5. Winkler, P. C.; Perkins, D. D.; Williams, W. K.; Browner, R. F. Anal. Chem. 1988, 60, 489-493.

6. Ashcroft, A. E. OMS Lett. 1987, 22, 754-757.

7. Caprioli, R. M. Biochemistry 1988, 27, 513-521.

8. Mosely, A. M.; Deterding, L. J.; Tomer, K. B.; Bragg, N.; Jorgenson, J. W. Capillary zone electrophoresis and capillary liquid chromatography coupled with sector mass spectrometry using coaxial continuous flow FAB interfaces, Proceedings of the 37th ASMS Conference on Mass Spectrometry and Allied Topics; Miami Beach, FL, May 21-26, 1989, p 114.

9. Bourell, J. H.; Henzel, W. J.; Stults, J. T. Analysis of enzymatically digested proteins by on-line capillary
HPLC/FABMS, presented at the 37th ASMS Conference on Mass Spectrometry and Allied Topics; Miami Beach, FL, May 21-26, 1989, p 1015.

10. Glajch, J. L. Anal. Chem. 1986, 58, 385-394.

11. Dong, M. W.; Gant, J. R.; Larsen, B. R. Biochromatography 1989, 4, 19-33.

12. Garnick, R. L.; Solli, N. J.; Papa, P. A. Anal. Chem. 1988, 60, 2546-2557.

13. Karas, M.; Hillenkamp, F. Anal. Chem. 1988, 60, 2301-2303.

14. Whitehouse, C. M.; Dreyer, R. N.; Yamashita, M.; Fenn, J. B. Anal. Chem. 1985, 57, 675-679.

15. Mann, M.; Meng, C. K.; Fenn, J. B. Anal. Chem. 1989, 61, 1702-1708.

16. Covey, T. R.; Bonner, R. F.; Shushan, B. I.; Henion, J. D. Rapid Commun. Mass Spectrom. 1988, 2, 249-255.

17. Henion, J. D.; Covey, T. R.; Muck, W.; Huang, E. C. LC/MS of proteins and large peptides. Presented at the 37th ASMS Conference on Mass Spectrometry and Allied Topics; Miami Beach, FL, May 21-26, 1989, p 1014.

18. Barinaga, C. J.; Edmonds, C. G.; Udseth, H. R.; Smith, R. D. Rapid Commun. Mass Spectrom. 1989, 3, 160-164.

19. Smith, R. D.; Loo, J. A.; Barinaga, C. J.; Udseth, H. Presented at the 5th (Montreux) Symposium on LC/MS, Freiburg, FRG, November 1988.

20. Schroeder, W. A. In Handbook of HPLC for the Separation of Amino Acids, Peptides, and Proteins; Hancock, W. S., Ed.; CRC: Boca Raton, FL, Vol. 2, Protein Identification by Peptide Mapping, 1984, pp 287-300.

21. Bruins, A. P.; Covey, T. R.; Henion, J. D. Anal. Chem. 1987, $59,2642-2646$.

22. Hattangadi, S. LC-GC 1989, 7, 108-114.

23. Lee, E. D.; Covey, T. R.; Henion, J. D. J. Microcol. Sep. 1989, $1,14-18$

24. Roepstorff, P.; Fohlman, J. Biomed. Mass Spectrom. 1984, 11, 601-602.

25. Hunt, D. F.; Zhu, N.; Shabanowitz, J.; Rapid Commun. Mass Spectrom. 1989, 3, 122-124.

26. Westmore, J. B.; Ens, W.; Standing, K. G. Biomed. Mass Spectrom. 1982, 9, 119-124.

27. Barber, M.; Bordoli, R. S.; Sedgwick, D.; Tyler, A. N.; Whalley, E. T. Biomed. Mass Spectrom. 1981, 8, 337-342.

28. Williams, D. H.; Bradley, C. V.; Santikarn, S.; Bojesen, G. J. Biochem. 1982, 201, 105-117.

29. Konig, W. A.; Aydin, M.; Schulze, U.; Rapp, U.; Hohn, M.; Pesch, R.; Kalikhevitch, V. N. Int. J. Mass Spectrom. Ion Phys. 1983, 46, 403-408.

30. Gaskell, S. J.; Haroldsen, P. E.; Reilly, M. H. Biomed. Mass Spectrom. 1988, 16, 31-33.

31. Heerma, W.; Kulik, W. Biomed. Mass Spectrom. 1988, 16, 155-159. 